prison such as Holloway Prison. Given the random process of admission and the often urgent need for skilled assessment and nursing, this unit must be on site, have a high staff to patient ratio as in psychiatric acute units or medium secure units, and have expertise in detoxification and withdrawal from drugs with links to outside services. Secondly, changes in the organisation of the psychiatric unit may well be required in structure, staffing, and philosophy of management, as recommended by the Holloway Project committee. ${ }^{17}$ Mentally disturbed women are constantly being admitted to Holloway Prison, often because there is nowhere else for them to go. Thus initiatives to alter policies of admission to psychiatric hospitals should be vigorously pursued. Finally, we think that a prison with its controlled environment is a suitable place for research, particularly into the treatment of personality disorders ${ }^{20}$ drug dependence, and deliberate cutting; any progress in these poorly understood topics of psychopathology would benefit not just the victims but society in general.

We thank the officers and nursing staff of Holloway Prison for their help; Dr P d'Orban for his advice and encouragement; and Drs Phillips and Berry for their support.

\section{References}

1 Smith R. Prison health care. London: British Medical Association, 1984.

2 Gibbens TCN. Female offenders. Br f Hosp Med 1971;6:279-90.

3 Home Office. Report on the work of the prison department 1982. London: HMSO, 1983.

4 Davies N. Protests grow over Holloway horror wing. Observer 1984 Sept 16:2.

5 Toynbee $P$. What shocks and horrifies is the sight of so many deeply deranged and disturbed women being in prison. Guardian 1984 Oct 15:10

6 Toynbee P. "Wrong" to send mentally ill to gaol. Guardian 1984 Oct $26: 3$.

7 Boseley S. Teacher quits to fight gaol regime. Guardian 1985 May 22:3.

8 Bowden P. Treatment and justice. Br f Hosp Med 1985;6:350-2.

9 Simpson MA. Self-mutilation. Br f Hosp Med 1976;10:430, 438

10 Carlen P. Women's imprisonment: a study in social control. London: Routledge and Kegan Paul, 1983.

11 d'Orban PT. Social and psychiatric aspects of female crime. Med Sci Law 1971;11:104-16.

12 Daniel AE, Harris PW, Husain SA. Differences between midlife female offenders and those younger than 40. Am f Psychiatry 1981;138:9,1225-8

13 Institute for the Study of Drug Dependence. Drug abuse briefing. London: Redesign, 1982.

14 Skegg DCG, Dell R, Perry J. Use of medicines in general practice. Br Med 7 1977;i:1561-3.

15 Coid J, Allolio B, Rees LH. Raised plasma metenkephalin in patients who habitually mutilate themselves. Lancet $1983 ;$ ii: $545-6$.

16 Cookson HM. A survey of self-injury in a closed prison for women. British Joumal of Criminology 1977; 17:332-47.

17 Home Office. Holloway project committee report. London: HMSO, 1985.

18 Office of Population Censuses and Surveys. OPCS monitor. London: HMSO, 1985. (GHS 85/2.)

19 Gunn J. Criminal behaviour and mental disorder. Br f Psychiatry 1977;130:317-29.

19 Gunn J. Criminal behaviour and mental disorder. Brf Psychiatry 1977;130:317-29.
20 Coid J. How many psychiatric patients in prison? Br f Psychiaty 1984;145:78-86.

(Accepted 6 fanuary 1986)

\title{
Six minute walking test for assessing exercise capacity in chronic heart failure
}

\author{
D P LIPKIN, A J SCRIVEN, T CRAKE, P A POOLE-WILSON
}

\begin{abstract}
Twenty six patients, mean age 58 years (range $36-68$ ), with stable chronic heart failure, New York Heart Association class II-III, and 10 normal subjects of a similar age range were studied. Exercise capacity was assessed by determining oxygen consumption reached during a maximal treadmill exercise test and by measuring the distance each patient walked in six minutes. There were significant differences in the distance walked in six minutes between normal subjects, patients with heart failure, class II, and those with class III heart failure $(683 \mathrm{~m}, 558 \mathrm{~m}$, and $402 \mathrm{~m}$, respectively $(p<0.003)$ ). The relation between maximal oxygen consumption and the distance walked in six minutes was curvilinear; thus the distance walked varied considerably in those with a low maximal oxygen consumption but varied little in patients and normal subjects with a high maximal oxygen consumption. All subjects preferred performing the six minute walking test to the treadmill exercise test, considering it to be more closely related to their daily physical activity.

The six minute test is a simple objective guide to disability in patients with chronic heart failure and could be of particular value in assessing patients with severe heart failure but less useful in assessing patients with mild heart failure.
\end{abstract}

Cardiothoracic Institute and National Heart Hospital, London WIN 2DX D P LIPKIN, BSC, MRCP, Squibb research fellow

A J SCRIVEN, BSC, MRCP, registrar

T CRAKE, MRCP, registrar

P A POOLE-WILSON, MD, FRCP, professor of cardiology

Correspondence and requests for reprints to: Professor Poole-Wilson.

\section{Introduction}

The severity of congestive heart failure is usually graded according to patients' reported symptoms, and in particular to the amount of physical activity that is associated with dyspnoea or fatigue. A more objective analysis would be useful to classify patients with congestive heart failure and to assess the efficacy of therapeutic interventions. Measurement of respiratory gas exchange provides a non-invasive assessment of maximal exercise capacity. ${ }^{1.5}$ Many patients, however, state that daily activities rarely entail the high workloads performed during a laboratory maximal exercise test. We investigated a simple practical test, the measurement of the distance walked in six minutes, ${ }^{6}$ to determine if this is a more realistic test of exercise capacity in patients with congestive heart failure.

\section{Patients and methods}

Twenty men and six women, mean age 58 (range 36-68), were studied. The cause of congestive heart failure was coronary artery disease in 12 , dilated cardiomyopathy in 10 , hypertensive heart disease in one, aortic regurgitation in two, and mitral regurgitation in one. There were 10 patients with New York Heart Association class II heart failure and 16 patients with New York Heart Association class III heart failure. All patients were taking diuretic treatment (mean dose: frusemide $42 \mathrm{mg}$ and amiloride $3 \mathrm{mg}$ for those with class II heart failure, frusemide $99 \mathrm{mg}$ and amiloride $12 \mathrm{mg}$ for those with class III heart failure). Other treatment included captopril $(n=8)$, nitrates $(n=4)$, and digoxin $(n=6$; all had atrial fibrillation). Exclusion criteria were myocardial infarction during the previous six months, evidence of myocardial ischaemia or ventricular tachycardia on exercise testing, obstructive valvular heart disease, pulmonary disease (noted by pulmonary function tests), or inability to exercise for any reason other than dyspnoea or fatigue. Ten normal subjects (seven men, three women) of similar age (range 36-62) were also studied and were found to be free of disease on the basis of history, physical examination, and 12 lead electrocardiography. All patients gave written informed consent to the study.

All subjects exercised twice to familiarise themselves with the treadmill before taking part in the study. Patients were exercised three hours after a 
light meal, at the same time of day, and at least three hours after any treatment. Each patient performed treadmill exercise using a modified Naughton protocol with low workloads increasing in six minute stages. ${ }^{3}$ Patients were encouraged to exercise until they were unable to continue. The exercise test was stopped at the patient's request. Ten normal subjects were exercised with the Bruce protocol to determine their maximal oxygen consumption. Maximal oxygen consumption was determined using an argon dilution technique and by mass spectrometric analysis of mixed expired air. ${ }^{7}$

The walking test was conducted as described by McGavin $e t a l,{ }^{8}$ but the timing was standardised to six minutes. The test was carried out in a level enclosed corridor $20 \mathrm{~m}$ long. Each patient was instructed to cover as much ground as possible in six minutes. Patients were told to walk continuously if possible but that they could slow down or stop if necessary. The aim was that at the end of the test the patients believed that they could not have walked any further in the six minutes. Patients were encouraged as necessary and advised when they had walked three and five minutes. The test was repeated twice on the same day with at least three to four hours between tests.

Results are expressed as mean (SEM), unless otherwise stated. Differences were analysed by analysis of variance and Student's $t$ test.

\section{Results}

Maximal oxygen consumption-Maximal oxygen consumption was limited by symptoms in all patients. No subject reached a plateau of oxygen consumption with increasing workload. ${ }^{9}$ Mean (SEM) maximal oxygen consumptions $(\mathrm{ml} / \mathrm{kg} / \mathrm{min})$ for normal subjects, patients with class II heart failure, and those with class III heart failure were $32(0 \cdot 8), 18(1 \cdot 2)$, and 12 $(0.4)$, respectively. There were significant differences in maximal oxygen consumption between normal subjects, patients with class II heart failure, and those with class III heart failure $(p<0.001)$ (table, fig 1 ).
Six minute walking test—For all subjects familiarisation resulted in a small increase in the distance walked in three successive tests: $396(125) \mathrm{m}$, 450 (126) $\mathrm{m}$, and 468 (120) $\mathrm{m}$, respectively. The mean (SEM) distances walked in the third test in normal subjects and in patients with class II and class III heart failure were $683(8) \mathrm{m}, 559(30) \mathrm{m}$, and $402(30) \mathrm{m}$ respectively. There were significant differences between the distances walked by normal subjects and by patients with classes II and III heart failure $(p<0 \cdot 003)$, though there was overlap between classes II and III (table, fig 2),

Maximal oxygen consumption was related in a curvilinear manner to the distance walked in all patients during the six minute walking test (fig 3 ). There was considerable variation in the distance walked $(390 \mathrm{~m}$, rang $144-540 \mathrm{~m})$ in patients with a low maximal oxygen consumption $(<12 \mathrm{ml} /$ $\mathrm{kg} / \mathrm{min})$. Conversely, the variation was less $(672 \mathrm{~m}$, range $630-720 \mathrm{~m})$ in those (patients and normal subjects) whose maximal oxygen consumption was greater than $20 \mathrm{ml} / \mathrm{kg} / \mathrm{min}$.

\section{Discussion}

In congestive heart failure the apparent severity of patients' symptoms may fluctuate widely according to mood and morale, although the patients' cardiac function may be unchanged. This variability suggests that symptoms are an unrealistic guide to the degree of physical limitation, yet clinicians often assess the severity of cardiac impairment largely on the basis of a clinical history.

Recent work in this and other laboratories has shown that exercise capacity can be objectively assessed by measuring peak oxygen consumption achieved with maximal bicycle or treadmill exercise. ${ }^{1.5}$ The equipment required to measure oxygen consumption is expensive, however, and some patients find treadmill exercise difficult.

Clinical characteristics, maximal oxygen consumption, and distance walked in six minutes in normal subjects and patients with congestive heart failure

\begin{tabular}{|c|c|c|c|c|c|c|c|}
\hline Case No & Age & Sex & Diagnosis & $\begin{array}{l}\text { Height } \\
(\mathrm{m})\end{array}$ & $\begin{array}{c}\text { Weight } \\
(\mathrm{kg})\end{array}$ & $\begin{array}{l}\text { Maximal oxygen } \\
\text { consumption } \\
(\mathrm{ml} / \mathrm{kg} / \mathrm{min})\end{array}$ & $\begin{array}{c}\text { Six minute } \\
\text { distance } \\
(\mathrm{m})\end{array}$ \\
\hline \multicolumn{8}{|c|}{ Normal subjects } \\
\hline 1 & 44 & $M$ & & $1 \cdot 70$ & 70 & 34 & 720 \\
\hline 2 & 36 & $\mathrm{M}$ & & 1.80 & 83 & 30 & 702 \\
\hline 3 & 44 & $\mathrm{M}$ & & 1.63 & 80 & 34 & 660 \\
\hline 4 & 50 & $M$ & & 1.55 & 65 & 31 & 666 \\
\hline 5 & 50 & $M$ & & 1.65 & 64 & 32 & 660 \\
\hline 6 & 47 & $\mathrm{M}$ & & 1.85 & 83 & 35 & 720 \\
\hline 7 & 62 & $\mathrm{M}$ & & 1.65 & 60 & 30 & 684 \\
\hline 8 & 46 & $\mathrm{~F}$ & & 1.64 & 54 & 34 & 659 \\
\hline 9 & 69 & $\mathrm{~F}$ & & 1.57 & 54 & 26 & 666 \\
\hline 10 & 48 & $M$ & & $1 \cdot 65$ & 65 & 35 & 700 \\
\hline Mean (SEM) & $49(3)$ & & & $1.66(0.03)$ & $68(3)$ & $31(1)$ & $683(8)$ \\
\hline \multicolumn{8}{|c|}{ Patients with NYHA class II heart failure } \\
\hline 11 & 61 & $M$ & IDC & $1 \cdot 76$ & 75 & 20 & 630 \\
\hline 12 & 60 & $M$ & Aortic regurgitation & 1.63 & 60 & 18 & 522 \\
\hline 13 & 55 & $\mathrm{~F}$ & IDC & 1.62 & 56 & 16 & 504 \\
\hline 14 & 61 & $M$ & CAD & 1.67 & 72 & 13 & 432 \\
\hline 15 & 50 & $\mathrm{~F}$ & Mitral regurgitation & 1.62 & 49 & 26 & 666 \\
\hline 16 & 18 & $\mathrm{~F}$ & IDC & 1.60 & 68 & 18 & 432 \\
\hline 17 & 49 & $M$ & CAD & 1.65 & 67 & 19 & 612 \\
\hline 18 & 64 & $\mathrm{M}$ & IDC & $1 \cdot 70$ & 70 & 17 & 606 \\
\hline 19 & 64 & $M$ & Aortic regurgitation & 1.70 & 63 & 15 & 650 \\
\hline 20 & 68 & $M$ & IDC & 1.65 & 65 & 19 & 540 \\
\hline Mean (SEM) & $53(4)$ & & & $1.65(0.01)$ & $64(2)$ & $18(1)$ & $562(30)$ \\
\hline \multicolumn{8}{|c|}{ Patients with NYHA class III heart failure } \\
\hline 21 & 63 & $\mathbf{M}$ & CAD & $1 \cdot 70$ & 64 & 14 & 558 \\
\hline 22 & 56 & $M$ & CAD & 1.64 & 64 & 11 & 450 \\
\hline 23 & 58 & $M$ & CAD & 1.69 & 66 & 12 & 468 \\
\hline 24 & 64 & $\mathrm{~F}$ & IDC & 1.47 & 45 & 13 & 360 \\
\hline 25 & 56 & $M$ & CAD & $1 \cdot 75$ & 88 & 10 & 414 \\
\hline 26 & 61 & $M$ & $\mathrm{CAD}$ & 1.62 & 56 & 10 & 396 \\
\hline 27 & 45 & $M$ & IDC & 1.76 & 86 & 14 & 504 \\
\hline 28 & 60 & $M$ & CAD & 1.76 & $\begin{array}{l}00 \\
88\end{array}$ & 11 & 216 \\
\hline 29 & 36 & $M$ & IDC & 1.65 & 71 & 11 & 516 \\
\hline 30 & 57 & $M$ & CAD & 1.65 & 82 & 9 & 360 \\
\hline 31 & 55 & $M$ & Hypertension & $1 \cdot 70$ & 106 & 12 & 504 \\
\hline 32 & 58 & $\mathrm{M}$ & CAD & 1.65 & 63 & 10 & 234 \\
\hline 33 & 66 & $M$ & IDC & 1.66 & 69 & 12 & 540 \\
\hline 34 & 59 & $M$ & IDC & 1.65 & 69 & 13 & 432 \\
\hline 35 & 65 & $\mathrm{~F}$ & CAD & 1.68 & 57 & 12 & 342 \\
\hline 36 & 64 & $\mathrm{~F}$ & CAD & $\begin{array}{l}1.60 \\
1.62\end{array}$ & 57 & 10 & 144 \\
\hline Mean (SEM) & $57(4)$ & & & $1.65(0.01)$ & $70(3)$ & $12(0 \cdot 4)$ & $402(30)$ \\
\hline
\end{tabular}

NYHA $=$ New York Heart Association, IDC $=$ idiopathic dilated cardiomyopathy, $\mathrm{CAD}=$ coronary artery disease 

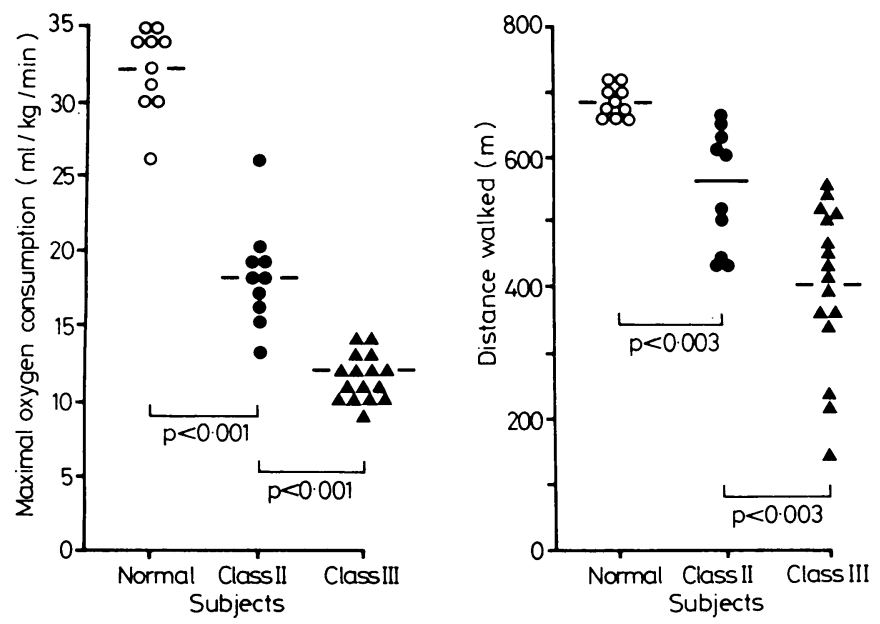

FIG 1-Relation between maximal oxygen consumption during six minute test and functional class in normal subjects and patients with New York Heart Association class II and III congestive heart failure.

FIG 2-Relation between distance walked during six minute test and functional class in normal subjects and patients with New York Heart Association class II and III congestive heart failure.

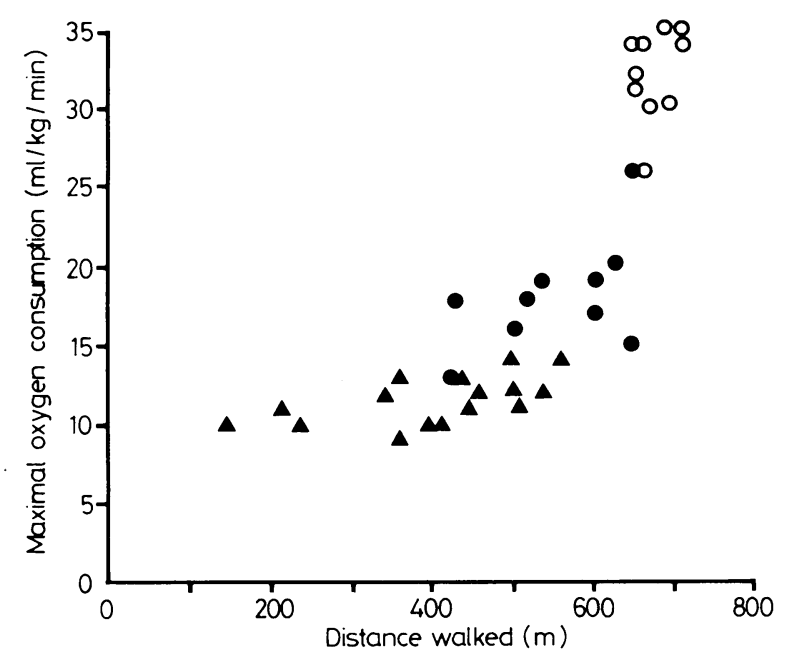

FIG 3-Relation between maximal oxygen consumption and distance walked during six minute test. Normal subjects $(\mathrm{O})$; patients with New York Heart Association class II heart failure $(\mathbf{)}$; patients with New York Heart Association class III heart failure $(\boldsymbol{\Delta})$.

The high workloads are not typical of the type of activity these patients perform in normal life.

We reasoned that in patients with moderate to severe disability it might be more appropriate to measure exercise performance at submaximal workloads. Various groups have used a two, six, and 12 minute walking test for normal subjects (12 minute running test $)^{10}$ and patients with chronic obstructive airways disease.$^{68} \mathrm{~A}$ walking test lasting six minutes has been suggested to be of the desired duration for assessing patients with severe disability. ${ }^{6}$ We are not aware of this test being used to assess patients with congestive heart failure.

Our results show that a simple six minute walking test is useful for objectively assessing exercise capacity in patients with congestive heart failure. All of our patients preferred the six minute walking test to the treadmill, finding it easier and more closely related to their daily physical activity. This may explain the wide range of distances covered in six minutes among those patients with a low maximal oxygen consumption. Some may have been poor performers on the treadmill and gave a better account of their abilities during the walking test.

Conversely, there was little variation in the distances covered in six minutes between normal subjects and patients with mild heart failure despite noticeable differences in their maximal exercise capacity. In these subjects the maximal rather than submaximal exercise test may be more suitable for assessing exercise capacity as exercise is performed at workloads sufficient to cause symptoms.

We conclude that in patients with congestive heart failure the six minute walk provides an objective assessment of exercise capacity that could usefully supplement clinical information obtained from the history and physical examination. The six minute test is less discriminating than the measurement of maximal oxygen consumption but has the advantage of being inexpensive and simple to perform. The test could be useful in assessing treatment in heart failure. It may be most suitable for serial monitoring of patients with moderate to severe heart failure, in whom repeated maximal exercise tests are unpleasant and unrealistic. Maximal exercise tests may be more appropriate for patients with mild heart failure.

We thank Claire Simpson, Sera Levinson, and Moira Craig of the physiotherapy department and Lesley Ludgate and Judith Hyland of the clinical measurement department at the National Heart Hospital for their help in performing this study.

\section{References}

1 Patterson JA, Naughton J, Pietras RJ, Gunnes RM. Treadmill exercise in the assessment of the functional capacity of patients with cardiac disease. Am $\mathcal{F}$ Cardiol 1972;30:757-62.

2 Weber KT, Kinasewitz GT, Janicki JS, Fishman AP. Oxygen utilization and ventilation during exercise in patients with chronic cardiac failure. Circulation 1982;65:1213-23.

3 Lipkin DP, Perrins J, Poole-Wilson PA. Respiratory gas exchange in the assessment of patients with impaired ventricular function. Br Heart $\mathcal{F}$ 1985;54:321-8.

4 Lipkin DP, Poole-Wilson PA. Measurement of cardiac output during exercise by the thermodilution and direct Fick techniques. Am 7 Cardiol 1985;56:321-4

5 Lipkin DP, Poole-Wilson PA. The ability of a submaximal exercise test to predict maximal exercise capacity in patients with heart failure. Eur Heart $\mathcal{f}$ 1985;6:829-33.

6 Butland RJA, Pang J, Gross ER, Woodcock AA, Geddes DM. Two-, six-, and 12-minute walking tests in respiratory disease. $\mathrm{Br}$ Med $\mathrm{J}$ 1982;284: 1607-8.

7 Davies N, Dennison D. Measurement of metabolic gas exchange and minute volume by mass spectron

spectrometry alone. Respir Physiol 1979;36:261-7.
8 McGavin CR, Gupta SP, Mettaidy GJR. Twelve minute walking test for assessing disability in chronic bronchitis. Br Med f 1976;1:822-3.

chronic bronchitis. Br.Med $\mathcal{F}$ 1976;1:822-3.
Taylor HL, Buskirk E, Henschel A. Maximal oxygen uptake as an objective measure of cardio9 Taylor HL, Buskirk E, Henschel A. Maximal oxygen 10 Cooper KH. A means of assessing maximal oxygen uptake. FAMA 1968;203:201-4.

(Accepted 14 January 1986) 